\title{
Canonical formalism for path-dependent Lagrangians. Coupling constant expansions
}

\author{
$X$. Jaén, R. Jáuregui, ${ }^{a)} \mathrm{J}$. Llosa, and A. Molina \\ Grup de Relativitat, Societat Catalana de Fisica (I.E.C.) and Departament Fisica Fonamental, Universitat \\ de Barcelona, Diagonal, 645, 08028-Barcelona, Spain
}

(Received 10 March 1989; accepted for publication 14 June 1989)

A canonical formalism obtained for path-dependent Lagrangians is applied to Fokker-type Lagrangians. The results are specialized for coupling constant expansions and later on are applied to relativistic systems of particles interacting through symmetric scalar and vector mesodynamics and electrodynamics.

\section{INTRODUCTION}

Hamiltonian formalism is a desirable feature to demand for a classical system. Indeed, it enables us to define the energy-momentum four-vector and the total angular momentum tensor as generating functions for Poincaré (resp. Galilei) infinitesimal transformations. Furthermore, it permits us to construct a statistical mechanics and a quantum mechanics according to some standard well defined rules.

On the other hand, relativistic dynamics for directly interacting particles, i.e., without an intermediate field, has been developed following a wide variety of approaches. ${ }^{1}$ In most of them a canonical formalism has been obtained, either because one a priori starts from a Hamiltonian system, ${ }^{2}$ or because an invariant symplectic form is obtained. ${ }^{3}$

However, there is an approach to relativistic action-ata-distance, that based on path-dependent Lagrangian systems, ${ }^{4-6}$ which has long refused a Hamiltonian formulation. ${ }^{7}$ This is especially striking since the starting point is a Lagrangian system and a variational principle, although of a very particular kind.

Path-dependent Lagrangians were first used by Fokker, ${ }^{4}$ who proposed an action principle for symmetric electrodynamics-half-retarded plus half-advanced-of two charges without an intermediate field. This is the reason why Lagrangians of this kind are also called "Fokker-type Lagrangians."

The symmetric electrodynamics of Feynman and Wheeler ${ }^{5,8}$ is a generalization of that of Fokker to the case of more than two charges.

Several other relativistic theories of noninstantaneous action-at-a-distance between particles have been set in terms of Fokker-type Lagrangians. ${ }^{6}$ This is usually the case of those interactions that are somehow related to a classical field.

Path-dependent Lagrangians exhibit a functional dependence on the trajectories as a whole. That makes these systems more complex than standard ones; but, since they permit us to consider interaction terms depending on noninstantaneous configurations of particles, these Lagrangians are especially useful for describing relativistic systems of directly interacting particles. The basic claim of these theories

\footnotetext{
a) Permanent address: Instituto de Física, Universidad Nacional Autonoma de México Apdo. Postal 20-364, México, 01000, D.F., Mexico.
}

is that the field is nothing but a useful tool to describe forces between particles. The concept of field, introduced by Faraday and Maxwell as an intermediate tool to describe the action of some given "source" and a "test charge," must be acknowledged as one of the most fruitful in theoretical physics. However, the self-interaction divergences in classical field theory occur as a result of allowing the field to act on its own source. In order to avoid this "improperty," some authors ${ }^{s}$ introduce what they call the "adjunct field." Namely, in Wheeler-Feynman symmetric electrodynamics, each charge is acted on by the "adjunct field" of the others, that is, half the sum of the advanced and retarded solutions of the Maxwell equations for the other charges. This leads to a Fokker-type Lagrangian, which only depends on particle variables.

The most important drawback of Fokker-type systems is that the Euler equations, derived from the Fokker action principle, are of functional-differential type (difference-differential equations in the simplest cases). Therefore, the evolution space (space of initial data) is non-Newtonian; that is, the positions and velocities in a given instant of "time" do not determine uniquely the future evolution of the system. Furthermore, the evolution space is not even well determined. ${ }^{7}$ As a consequence, it has not been possible to generalize an algorithm as a Legendre transformation to Fokkertype Lagrangians, nor has an equivalent Hamiltonian formalism been set up yet. We can overcome this problem ${ }^{9}$ by changing the point of view that is usual in classical mechanics. Since the Euler equations derived from a path-dependent Lagrangian are of functional-differential type, the initial data space for a Fokker Lagrangian has infinitely many dimensions. In our approach, a whole trajectory of the system is taken as the "initial datum." In doing this, the Euler equations do not rule the evolution anymore (all information about it is already contained in the initial datum), and they are merely considered as constraints on the initial data.

This approach somehow corresponds to a static point of view. The situation is similar to what happens in dealing with a static standard Lagrangian $L(q, t)$ (i.e., one depending on coordinates only): the initial data $\left(q_{0 a}\right)_{a=1, \ldots, m}$ can, in principle, be picked out from an $m$-dimensional continuum, but the physically significant ones are only those satisfying 


$$
\left(\frac{\partial L}{\partial q_{a}}\right)_{q_{0} \ldots, \ldots, q_{0 m}}=0 \text {. }
$$

Similarly, in our approach to path-dependent Lagrangian systems, one can, in principle, take any $m$-tuple of curves $\left[q_{1}\left(t_{1}\right), \ldots, q_{m}\left(t_{m}\right)\right]$ as initial data, but these will be physically significant only if we have been lucky enough to have chosen a set of curves fulfilling the Euler functionaldifferential equations, now considered as constraints.

As has been mentioned elsewhere, those functional differential equations admit "too many solutions": contrary to what we are used to in Newtonian mechanics, the knowledge of all positions and velocities at a given time does not determine the future evolution of the system. This is a feature of Newtonian physics that seems worthy to preserve, as many authors $^{10,11}$ have considered. Hence several criteria have been put forward in order to get rid of the "physically irrelevant" solutions. Among these criteria, it is worth mentioning that (a) when coupling constants go to zero, motions must become free (namely, uniform and rectilinear) ${ }^{12-14}$; (b) in the limit $1 / c^{2} \rightarrow 0$, the solutions must yield the Newtonian ones ${ }^{11,15,16}$; and (c) if one mass is much bigger than than the others, then the external field approximation must be recovered. ${ }^{17.18}$

Any of these three criteria is implemented by requiring the physically relevant solutions to be analytical in the corresponding parameter, namely, the coupling constants, the inverse speed of light, or the mass ratio, respectively. Any of them selects a family of solutions of the functional-differential equations parametrized by either $6 N$ (noncovariant formalism) or $8 N$ (covariant formalism) parameters. It then happens (apparently as a consequence of the special structure of the Fokker-type Lagrangian) that these solutions also satisfy a second-order differential system that can be obtained by techniques ${ }^{19}$ provided by predictive relativistic mechanics. This differential system is called a second-order reduction ${ }^{19 \mathrm{a}}$ of the functional-differential system.

So that, in the covariant formalism given in Ref. 9, we consider the map

$$
\begin{aligned}
\varphi: & \mathrm{TM}_{4}^{N} \rightarrow E, \\
& (x, \dot{x}) \rightarrow \varphi_{a}^{\mu}\left(\xi, x_{b}, \dot{x}_{c}\right),
\end{aligned}
$$

where $\varphi_{a}^{\mu}$ is the predictive solution of the functional-differential system determined by one of the above-mentioned criteria and the initial datum $\left(x_{b}, \dot{x}_{c}\right)$.

Then, using a kind of Ostrogradski transformation, we set up a Hamiltonian formalism for path-dependent Lagrangians (Sec. II). Once this has been done for Fokker-type Lagrangian systems, as a particular case of path-dependent Lagrangians, it can be specialized to any reduction of order of the Fokker system.

The paper is organized as follows: in Sec. II we give the canonical formalism for Fokker-type systems. In Sec. III, we derive a presymplectic form on the infinite-dimensional evolution space $E$ of a Fokker-type system of relativistic particles with two-body interactions. Then (Sec. IV) this presymplectic form is specialized to the Newton-like evolution space $\mathrm{TM}_{4}^{N}$, which results from implementing the condition of analytical dependence on the coupling constant. In the latter, we briefly describe perturbation theory and introduce the three-dimensional formalism.

The results obtained are then applied to scalar and vector interactions, up to the first order of approximation in the product $g_{a} g_{b}$ of the coupling constants. Special attention is paid to Wheeler-Feynman electrodynamics, for which expansions on $1 / c^{2}$ are carried out and conserved quantities are also calculated.

\section{HAMILTONIAN FORMALISM FOR FOKKER LAGRANGIANS}

Let us consider a Fokker Lagrangian

$$
\begin{aligned}
L= & -\sum_{a=1}^{N} m_{a}\left(-\dot{x}_{a}^{2}(t)\right)^{1 / 2} \\
& -\sum_{\substack{a, b=1 \\
a \neq b}}^{N} g_{a} g_{b} \int_{\mathbf{R}} d \xi \omega_{a b}^{r}(t, t+\xi),
\end{aligned}
$$

where

$\omega_{a b}^{r}\left(t_{a}, t_{b}\right)=G_{a b}\left(\left(x_{a}\left(t_{a}\right)-x_{b}\left(t_{b}\right)\right)^{2}\right) F_{a b}\left(\dot{x}_{a}\left(t_{a}\right), \dot{x}_{b}\left(t_{b}\right)\right)$

and

$F_{a b}\left(\dot{x}_{a}, \dot{x}_{b}\right)=\left(-\dot{x}_{a} \dot{x}_{b}\right)^{r}\left(-\dot{x}_{a}^{2}\right)^{(1-r) / 2}\left(-\dot{x}_{b}^{2}\right)^{(1-r) / 2}$,

$a^{2}=a_{\mu} a^{\mu}$, for any four-vector $a_{\mu}$, and $r \in N$ depends on the specific interaction we are considering.

Notice that $L$ is a homogeneous function of first degree of velocities. Therefore, the corresponding action integral is reparametrization invariant. We also notice that

$\omega_{a b}^{r}\left(t_{a}, t_{b}\right)=\omega_{b a}^{r}\left(t_{b}, t_{a}\right)$.

This Fokker Lagrangian can be put in the form

$$
\begin{aligned}
L_{t}= & \int_{\mathbf{R}^{m}} d \xi_{1}, \ldots, d \xi_{m} \mathscr{L}\left(q_{a}\left(t+\xi_{a}\right), \dot{q}_{b}\left(t+\xi_{b}\right), \xi_{c}\right), \\
& a, b, c=1, \ldots, m,
\end{aligned}
$$

by merely taking

$$
\mathscr{L}=\mathscr{L}_{0}+\mathscr{L}_{\mathrm{I}}
$$

with

$$
\begin{aligned}
& \mathscr{L}_{0}=-\sum_{a=1}^{N} m_{a}\left(-\dot{x}_{a}^{2}\left(t+\xi_{a}\right)\right)^{1 / 2} \prod_{d=1}^{N} \delta\left(\xi_{d}\right), \\
& \mathscr{L}_{\mathrm{I}}=-\frac{1}{2} \sum_{\substack{a, b=1 \\
a \neq b}}^{N} g_{a} g_{b} \omega_{a b}^{r}\left(t+\xi_{a}, t+\xi_{b}\right) \prod_{d=1}^{N} \delta\left(\xi_{d}\right) .
\end{aligned}
$$

In Ref. 9 we showed how a canonical formalism can be obtained for this kind of Lagrangian (2.5).

The procedure can be briefly described as follows. We start from the action $S=\int L_{t} d t$ and the variational principle $\delta S=0$, where the variations $\delta q_{a}\left(\xi_{a}\right)$ are taken so that they have compact support, gives rise to the equations of functional type

$$
\int_{\mathbf{R}} d \xi\left[f_{a}(\tau-\xi, \xi)-\frac{\partial}{\partial \tau} g_{a}(\tau-\xi, \xi)\right]=0
$$


where

$$
f_{a}\left(\tau, \xi_{a}\right)=\int_{\mathbf{R}^{m-1}} \prod_{b \neq a} d \xi_{b} \frac{\partial \mathscr{L}}{\partial q_{a}\left(\tau+\xi_{a}\right)}
$$

and

$$
g_{a}\left(\tau, \xi_{a}\right)=\int_{\mathbb{R}^{m-1}} \prod_{b \neq a} d \xi_{b} \frac{\partial \mathscr{L}}{\partial \dot{q}_{a}\left(\tau+\xi_{a}\right)} .
$$

However, because of the functional-differential character of the equations of motion, it is necessary to clarify their meaning. In principle, the initial data that should be given to specify a unique solution are the whole functions $q_{a}(\lambda)$ themselves. As a consequence, these equations [ (2.6)] must be regarded as constraints defining the evolution space $E$ (also called initial data space), rather than as laws of motion.

We then consider the Hamiltonian

$$
H=\sum_{a=1}^{m} \int_{\mathbf{R}} d \lambda p_{a}(\lambda) \dot{q}_{a}(\lambda)-L_{0}\left[q_{b}\left(\xi_{b}\right)\right]
$$

defined on a phase space $\Gamma$ labeled by

$$
q_{b}\left(\xi_{b}\right), p_{a}\left(\xi_{a}\right), \quad a, b=1, \ldots, m, \quad \xi_{a}, \xi_{b} \in \mathbb{R},
$$

with elementary Poisson brackets

$$
\begin{aligned}
& \left\{q_{a}(\xi), p_{b}\left(\xi^{\prime}\right)\right\}=\delta_{a b} \delta\left(\xi-\xi^{\prime}\right), \\
& \left\{q_{a}(\xi), q_{b}\left(\xi^{\prime}\right)\right\}=\left\{p_{a}(\xi), p_{b}\left(\xi^{\prime}\right)\right\}=0 .
\end{aligned}
$$

We then introduce the primary constraints

$$
\begin{aligned}
p_{a}(\lambda)= & g_{a}(0, \lambda)+\int_{\mathbf{R}} d \xi\left[f_{a}(\lambda-\xi, \xi)\right. \\
& \left.-\partial_{\lambda} g_{a}(\lambda-\xi, \xi)\right] \Theta(\lambda-\xi, \xi),
\end{aligned}
$$

with

$$
\begin{aligned}
\Theta(u, v) & =Y(v) Y(u)-Y(-v) Y(u) \\
& =\frac{1}{2}[\varepsilon(v)-\varepsilon(u)],
\end{aligned}
$$

where $Y(v)$ denotes the Heaviside function and $\varepsilon(v)$ is the "sign function." In Ref. 9, it was found that the secondary constraints that follow from (2.11) and the Hamiltonian (2.8) are the functional equations (2.6). Therefore, the evolution space $E$ can be immersed into the phase space $\Gamma$ by defining

$$
\begin{aligned}
& \psi: E \rightarrow \Gamma, \\
& z=q_{a}\left(\lambda_{a}\right) \rightarrow \psi(z)=\left(q_{a}\left(\lambda_{a}\right), p_{b}\left(\lambda_{b}\right)\right),
\end{aligned}
$$

where each curve $p_{b}\left(\lambda_{b}\right)$ is given as a function of $z=\left(q_{a}\left(\lambda_{a}\right)\right)$ by Eq. (2.11). This mapping is invariant under "time" $t$ evolution. Then, the symplectic form defined on $\Gamma$ by the Poisson brackets (2.10) can be pulled back onto $E$. In this way, an equivalence between the Lagrangian formalism (2.6) and (2.7) and the Hamiltonian one (1.5)-(1.9) is established.

\section{PRESYMPLECTIC FORM ON E}

If we apply the above results to Fokker Lagrangians we obtain

$$
\begin{aligned}
f_{a \mu}(\lambda, \xi)= & -\frac{1}{2} \sum_{b \neq a} g_{a} g_{b} \\
& \times \int_{\mathbf{R}} d \eta \frac{\partial \omega_{b a}^{r}(\lambda+\eta, \lambda+\xi)}{\partial x_{a}^{\mu}(\lambda+\xi)}[\delta(\xi)+\delta(\eta)]
\end{aligned}
$$

and

$$
\begin{aligned}
g_{a \mu}(\lambda, \xi)= & \delta(\xi) m_{a}\left(-\left(\dot{x}_{a}^{2}(\lambda)\right)^{-1 / 2} \dot{x}_{a \mu}(\lambda)\right. \\
& -\frac{1}{2} \sum_{\substack{b=1 \\
b \neq a}}^{N} g_{a} g_{b} \int_{\mathbf{R}} d \eta \\
& \times \frac{\partial \omega_{b a}^{r}(\lambda+\eta, \lambda+\xi)}{\partial \dot{x}_{a}^{\mu}(\lambda+\xi)}[\delta(\xi)+\delta(\eta)] .
\end{aligned}
$$

As a consequence, the Euler equations take the form

$$
\begin{aligned}
\frac{d}{d \lambda} & \left(m_{a} \dot{x}_{a \mu}(\lambda)\left(-\dot{x}_{a}^{2}(\lambda)\right)^{-1 / 2}\right) \\
& =\sum_{\substack{b=1 \\
b \neq a}}^{N} g_{a} g_{b} \int_{\mathbf{R}} d \eta \hat{\mathscr{L}}_{a \mu} \omega_{a b}^{r}(\lambda-\eta, \lambda),
\end{aligned}
$$

with the Lagrange operators $\hat{\mathscr{L}}_{a \mu}$ defined by

$$
\hat{\mathscr{L}}_{a \mu}=\frac{\partial}{\partial x_{a}^{\mu}(\lambda)}-\frac{d}{d \lambda} \frac{\partial}{\partial \dot{x}_{a}^{\mu}(\lambda)} .
$$

The primary constraints $(2.11)$ can be written as

$$
p_{a \mu}(\lambda)=\delta(\lambda) \pi_{a \mu}+\eta_{a \mu}(\lambda),
$$

where

$$
\begin{aligned}
\pi_{a \mu}= & m_{a}\left(-\dot{x}_{a}^{2}(0)\right)^{-1 / 2} \dot{x}_{a \mu}(0) \\
& -\sum_{\substack{b=1 \\
b \neq a}}^{N} g_{a} g_{b} \int_{\mathbb{R}} d \eta \frac{\partial w_{a b}^{r}(\eta, 0)}{\partial \dot{x}_{a}^{\mu}(0)}
\end{aligned}
$$

and

$$
\begin{aligned}
\eta_{a \mu}(\lambda)= & -\frac{1}{2} \sum_{\substack{b=1 \\
b \neq a}}^{N} g_{a} g_{b} \\
& \times \int_{\mathbb{R}} d \eta \hat{\mathscr{L}}_{a \mu}\left\{\omega_{a b}^{r}(\lambda-\eta, \lambda)\right\} \Theta(\lambda-\eta, \lambda) .
\end{aligned}
$$

Notice that the term $\pi_{a \mu}$ looks like the expression obtained for mechanical momenta in an analogous field description of the system.

The phase space $T^{*} E$ was endowed with a Liouville form $\Xi \in \Lambda^{1}\left(T^{*} E\right)$, and the corresponding symplectic structure $^{9}$

$$
\Omega=-d \Xi \text {. }
$$

The pullback map $\psi^{*}$ takes $\Xi \in \Lambda^{1}\left(T^{*} E\right)$ onto $\psi^{*} \Xi \in \Lambda^{1}(E)$; thus yielding

$$
\psi^{*} \Xi=\sum_{a=1}^{N} \int_{\mathbf{R}} d \lambda p_{a}^{\mu}\left(\lambda,\left[x_{a}(\lambda)\right]\right) \Delta x_{a \mu}(\lambda) .
$$

Here the symbol $\Delta$ denotes the exterior differential in the infinite-dimensional manifold $E$, and it is used in order to avoid confusion with the symbol $d \lambda$ under the integral sign. Substituting Eq. (2.5) into (2.8) we directly obtain 


$$
\begin{aligned}
\psi^{*} \Xi= & \sum_{a=1}^{N} \pi_{a}^{\mu} \Delta x_{a \mu}(0) \\
& +\sum_{a=1}^{N} \int_{\mathbf{R}} d \lambda \eta_{a}^{\mu}(\lambda) \Delta x_{a \mu}(\lambda)
\end{aligned}
$$

Notice the simplicity of the first term compared with the second one, which involves an integral over the region of $\mathbb{R}^{2}$ having $\Theta(\lambda-\eta, \lambda)$ as its characteristic function.

The expressions for the generating functions of the Poincaré group can be directly obtained, too, as we already discussed in Ref. 9. Thus the total linear momentum is given by the generating functions of space-time translations:

$$
\begin{aligned}
P_{\mu} & =\sum_{a=1}^{N} \int_{\mathbf{R}} d \lambda p_{a \mu}(\lambda) \\
& =\sum_{a=1}^{N} \pi_{a \mu}+\sum_{a=1}^{N} \int_{\mathbf{R}} d \lambda \eta_{a \mu}(\lambda) .
\end{aligned}
$$

While the generating functions for Lorentz transformations yield the total angular momentum ${ }^{9}$

$$
\begin{aligned}
J_{\mu \nu}= & \sum_{a=1}^{N} \int_{\mathbf{R}} d \lambda\left\{x_{a \mu}(\lambda) p_{a v}(\lambda)-x_{a v}(\lambda) p_{a \mu}(\lambda)\right\} \\
= & \sum_{a=1}^{N}\left\{x_{a \mu}(0) \pi_{a v}-x_{a v}(0) \pi_{a \mu}\right\} \\
& +\sum_{a=1}^{N} \int_{\mathbf{R}} d \lambda\left\{x_{a \mu}(\lambda) \eta_{a v}(\lambda)-x_{a v}(\lambda) \eta_{a \mu}(\lambda)\right\}
\end{aligned}
$$

These results are in agreement with earlier calculations obtained, either by a generalization of the Noether theorem ${ }^{7}$ or by the method of Dettman and Schild, ${ }^{20}$ provided that

$$
\lim _{\lambda \rightarrow \infty} \omega_{a b}^{r}(\lambda, \eta)=0 .
$$

\section{THE PRESYMPLECTIC STRUCTURE ON TMU}

Let us consider a set of solutions of Eqs. (3.3),

$x_{a}^{\mu}(\lambda)=\varphi_{a}^{\mu}\left(\lambda, x_{b}, \dot{x}_{c}\right)$,

parametrized with the Newton-like initial data $x_{b}^{\nu}(0)=x_{b}^{\nu}$, $\dot{x}_{c}^{\rho}(0)=\dot{x}_{c}^{\rho}$.

When we introduce them in Eq. (3.9) we obtain

$$
\begin{aligned}
\rho= & j^{*} \Xi \\
= & \sum_{a=1}^{N} \pi_{a \mu}\left(x_{b}, \dot{x}_{c}\right) \Delta x_{a}^{\mu} \\
& +\sum_{a=1}^{N} \int_{\mathbb{R}} d \lambda \eta_{a \mu}\left(\lambda, x_{b}, \dot{x}_{c}\right) \Delta \varphi_{a}^{\mu}\left(\lambda, x_{b}, \dot{x}_{c}\right),
\end{aligned}
$$

where $j^{*}=\varphi^{* \circ} \psi^{*}{ }^{9}$ Moreover, $\pi_{a \mu}\left(x_{b}, \dot{x}_{c}\right)$ and $\eta_{a \mu}\left(x_{b}, \dot{x}_{c}\right)$ correspond to $\pi_{a \mu}$ and $\eta_{a \mu}$ evaluated by introducing (4.1) into (3.5), and use has been made of the fact that

$$
\begin{aligned}
j^{*}\left(\Delta x_{a}^{\mu}(\lambda)\right) & =\Delta \varphi_{a}^{\mu}\left(\lambda, x_{b}, \dot{x}_{c}\right) \\
& \equiv \sum_{b=1}^{N} \frac{\partial \varphi_{a}^{\mu}}{\partial x_{b}^{v}} d x_{b}^{v}+\sum_{b=1}^{N} \frac{\partial \varphi_{a}^{\mu}}{\partial \dot{x}_{b}^{v}} d \dot{x}_{b}^{v}
\end{aligned}
$$

and

$$
\varphi_{a}^{\mu}\left(0, x_{b}, \dot{x}_{c}\right)=x_{a}^{\mu} .
$$

In this way, we obtain a formal expression for the Liouville form on $\mathrm{TM}_{4}^{N}$. An analogous treatment can be given to the total linear and angular momenta. Now, actual calculations require explicit expressions for the trajectories $\varphi_{a}^{\mu}$, $a=1, \ldots, N$. As we have already mentioned in the Introduction, these can be dealt with through perturbative techniques, which can be found in the literature. ${ }^{19}$ These techniques use the analytical dependence on the physical parameters that we have assumed for the solutions $\varphi_{a}^{\mu}$. Then, the corresponding MacLaurin series on these parameters are worked out and a perturbative expansion is obtained. In the present paper, we mainly work with coupling constant expansions. Let us briefly describe the associated perturbation theory.

Consider the expansion of $x_{a \mu}$ in powers of the coupling constants $g_{a}$ :

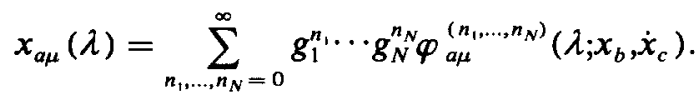

When (4.4) is substituted into the equations of motion (3.3), a hierarchy of second-order differential systems is obtained, such that each step determines the right-hand side of the next one. The condition that in the limit $g_{a} \rightarrow 0$ we have free motion is then introduced by taking

$$
\varphi_{a \mu}^{(0)}\left(\lambda ; x_{b}, \dot{x}_{c}\right)=x_{a \mu}+\lambda \dot{x}_{a \mu}
$$

and the coefficients in the series (4.4) are uniquely determined by the Newton-like set of initial data $\left(x_{b}, \dot{x}_{c}\right)$ at $\lambda=0$.

A similar treatment could be used for the problem if the criteria (b) or (c) discussed in Sec. I were chosen, namely, $1 / c^{2}$ (see Refs. 11,15 , and 16 ) and $m / M$ (see Refs. 17 and 18) expansions, respectively.

Introducing these kinds of expansions in (4.2) we obtain a perturbative expansion for $\rho$. Furthermore, since the lowest-order term in the expansion for the Lagrange operator $\hat{\mathscr{L}}_{a \mu}$ is given by

$$
\hat{\mathscr{L}}_{a \mu}^{(0)}=\frac{\partial}{\partial x^{a \mu}}-\frac{\partial}{\partial \lambda}\left(\frac{\partial}{\partial \dot{x}^{a \mu}}-\lambda \frac{\partial}{\partial x^{a \mu}}\right),
$$

the order of computing integrals and derivatives in Eq. (2.13) can be reversed.

We also have that

$$
\Delta \varphi_{a \mu}^{(0)}\left(\lambda ; x_{b}, \dot{x}_{c}\right)=d x_{a \mu}+\lambda d \dot{x}_{a \mu} .
$$

And, after some rearrangements, it is found that, to the order $n=2$,

$$
\rho=\sum_{a=1}^{N}\left(R_{a \mu}^{(2)} d x_{a}^{\mu}+Q_{a \mu}^{(2)} d \dot{x}_{a}^{\mu}\right)+O\left(g^{4}\right),
$$

where the term $O\left(g^{n}\right)$ includes all terms occurring multiplied by at least $n$ "charges" $g_{b}$. Moreover,

$$
\begin{aligned}
R_{a \mu}^{(2)}= & \pi_{a \mu}^{(2)}(x, \dot{x}) \\
& -\frac{1}{2} \sum_{b \neq a} g_{a} g_{b}\left[F_{b a}\left(\dot{x}_{b}, \dot{x}_{a}\right) \frac{\partial}{\partial x_{a}^{\mu}}\left(N_{b a}^{0}+M_{b a}^{1}\right)\right. \\
& \left.-\frac{\partial}{\partial \dot{x}_{a}^{\mu}}\left(F_{b a}\left(\dot{x}_{b}, \dot{x}_{a}\right) M_{b a}^{0}\right)\right]
\end{aligned}
$$




$$
\begin{aligned}
Q_{a \mu}^{(2)}= & -\frac{1}{2} \sum_{b \neq a} g_{a} g_{b}\left[F_{b a}\left(\dot{x}_{b}, \dot{x}_{a}\right) \frac{\partial M_{b a}^{2}}{\partial x_{a}^{\mu}}\right. \\
& \left.+\frac{\partial}{\partial \dot{x}_{a}^{\mu}}\left[\left(N_{b a}^{0}-M_{b a}^{1}\right) F_{b a}\left(\dot{x}_{b}, \dot{x}_{a}\right)\right]\right],
\end{aligned}
$$

$F_{b a}\left(\dot{x}_{b}, \dot{x}_{a}\right)$ is given by (2.3), and

$$
\begin{aligned}
& N_{b a}^{s}=\int_{\mathbf{R}^{2}} d \lambda d \eta \lambda^{s} \Theta(\lambda-\eta, \lambda) G_{b a}^{(0)}(\lambda-\eta, \lambda), \\
& M_{b a}^{s}=\int_{\mathbb{R}^{2}} d \lambda d \eta \partial_{\lambda}\left[\lambda^{s} \Theta(\lambda-\eta, \lambda) G_{b a}^{(0)}(\lambda-\eta, \lambda)\right],
\end{aligned}
$$

where, according to (4.5),

$$
G_{b a}^{(0)}\left(\lambda_{b}, \lambda_{a}\right)=G_{b a}\left(x_{b}-x_{a}+\dot{x}_{b} \lambda_{b}-\dot{x}_{a} \lambda_{a}\right) .
$$

Similarly, to this order of approximation, the total linear momentum is given by

$$
P_{\mu}=\sum_{a=1}^{N} R_{a \mu}^{(2)}+O\left(g^{4}\right),
$$

while the total angular momentum is

$$
\begin{aligned}
J_{\mu \nu}= & \sum_{a=1}^{N}\left[R_{a \mu}^{(2)} x_{a v}+Q_{a \mu}^{(2)} \dot{x}_{a v}\right. \\
& \left.-R_{a v}^{(2)} x_{a \mu}-Q_{a v}^{(2)} \dot{x}_{a \mu}\right]+O\left(g^{4}\right) .
\end{aligned}
$$

Hitherto, we have been working in the covariant formalism. The price we have had to pay for this is the singular character of the lowest-order term in the Lagrangian. As a consequence, the lowest-order contribution to the presymplectic form on $\mathrm{TM}_{4}^{N}$ is not regular. In other words, the zeroth-order terms in the momenta (3.6a), $\pi_{a \mu}^{(0)}\left(x_{b}, \dot{x}_{c}\right)$, fulfill the $N$ constraints

$$
\pi_{a}^{(0) \mu} \pi_{a \mu}^{(0)}\left(x_{b}, \dot{x}_{c}\right)=-m_{a}^{2} .
$$

In order to avoid the problems associated with this singularity, we shall go into the noncovariant formalism by fixing the time coordinates $x_{a}^{0}$ and the evolution parameter $t$ according to

$$
x_{a}^{0}=t .
$$

Given any quantity $A$ in the covariant formalism, we shall denote by $\widetilde{A}$ its noncovariant counterpart, that is, the quantity resulting from introducing the constraints (4.13) into it.]

A direct calculation shows that

$$
\tilde{\rho}=H d t-\sum_{a=1}^{N} \vec{p}_{a} d \vec{q}_{a},
$$

where

$$
\begin{aligned}
& H=-\sum_{a=1}^{N} \widetilde{R}_{a \infty}^{(2)}+O\left(g^{4}\right) \\
& \vec{q}_{a}=\vec{x}_{a}-\frac{1}{m_{a} \gamma_{a}}\left[\overline{\vec{Q}}_{a}^{(2)}-\left(\overrightarrow{\vec{Q}}_{a}^{(2)} \vec{v}_{a}\right) \vec{v}_{a}\right]+O\left(g^{4}\right),
\end{aligned}
$$

and

$$
\vec{p}_{a}=\tilde{\vec{R}}_{a}^{(2)}+O\left(g^{4}\right) .
$$

The one-form $\tilde{\rho}$ obtained by restriction of (4.8) to the phase space defined by the constraints (4.13) (that is, the phase space of the noncovariant formalism) plays the role of a Poincaré-Cartan integral invariant. ${ }^{21}$ According to this $H$ will be the Hamiltonian, i.e., the generating function for time evolution, and $\vec{q}_{a}$ and $\vec{p}_{a}$ will be a set of canonical coordinates and momenta. (As usual, an arrow over a symbol indicates the space part of the corresponding four-yector.)

The evaluation of the quantities $\vec{R}_{a}^{(2)}$ and $\vec{Q}_{a}^{(2)}$ involves differentiation with respect to $x_{a}^{\mu}$ and $\dot{x}_{a}^{\mu}$. (Some useful formulas are given in the Appendix.)

\section{SCALAR AND VECTOR INTERACTIONS. WHEELER- FEYNMAN PREDICTIVE ELECTRODYNAMICS}

\section{A. Scalar and vector interactions}

The action-at-a-distance counterpart of the field theory for scalar particles interacting through a massive scalar (resp. vector) field is given by the Lagrangian (2.1) with $r=0($ resp. $r=1)$ and

$G_{a b}\left(\left(x_{a}\left(t_{a}\right)-x_{b}\left(t_{b}\right)\right)^{2}\right)=D_{\mathrm{sym}}\left(\mu,\left(x_{a}\left(t_{a}\right)-x_{b}\left(t_{b}\right)\right)^{2}\right)$,

where

$$
\begin{aligned}
D_{\text {sym }}(\mu, s) & =\frac{1}{2}\left[D_{\text {Adv }}(\mu, s)+D_{\text {ret }}(\mu, s)\right] \\
& =2\left[\delta(s)-Y(-s)(\mu / 2 \sqrt{-s}) J_{1}(-\mu \sqrt{-s})\right]
\end{aligned}
$$

is the time symmetric Green's function for the massive Klein-Gordon operator.

In order to obtain a presymplectic structure on $\mathrm{TM}_{4}^{N}$, up to the second order in the coupling constants $g_{a}$, it is necessary to give explicit expressions for the results (4.10). First of all, we notice that, up to this order,

$$
\begin{aligned}
F_{a b}\left(\dot{x}_{a}, \dot{x}_{b}\right)= & \left(-\dot{x}_{a} \dot{x}_{b}\right)^{r}\left(-\dot{x}_{a} \dot{x}_{a}\right)^{(1-r) / 2} \\
& \times\left(-\dot{x}_{b} \dot{x}_{b}\right)^{(1-r) / 2},
\end{aligned}
$$

and therefore it does not depend on $\lambda$.

Substituting (5.1) into Eq. (4.10b), we also have

$$
\begin{gathered}
M_{a b}^{s}=2 \int d \lambda d \eta \partial_{\lambda}\left[\lambda^{s} \Theta(\lambda-\eta, \lambda) D_{\mathrm{sym}}\left(\mu, f_{a b}(\lambda, \eta)\right)\right] \\
\begin{aligned}
f_{a b}(\lambda, \eta) & =\left[\left(x_{a}-x_{b}\right)+\left(\dot{x}_{a}-\dot{x}_{b}\right) \lambda-\dot{x}_{b} \eta\right]^{2} \\
& \equiv\left[x_{a b}+\dot{x}_{a b} \lambda-\dot{x}_{b} \eta\right]^{2}
\end{aligned}
\end{gathered}
$$

Then, taking into account that

$$
\underset{x \rightarrow \infty}{J_{1}(x) \rightarrow 1 / \sqrt{x} \rightarrow 0,}
$$

we obtain

$$
M_{a b}^{s}=0, \quad s=0,1,2 .
$$

Consequently, Eqs. (4.10) take the simpler form

$$
\begin{aligned}
& R_{a \mu}^{(2)}=\pi_{a \mu}^{(2)}-\frac{1}{2} \sum_{b \neq a} g_{a} g_{b} F_{b a}\left(\dot{x}_{b}, \dot{x}_{a}\right) \frac{\partial}{\partial x_{a}^{\mu}} N_{b a}^{0}, \\
& Q_{a \mu}^{(2)}=-\frac{1}{2} \sum_{b \neq a} g_{a} g_{b} \frac{\partial}{\partial \dot{x}_{a}^{\mu}}\left(N_{b a}^{0} F_{b a}\left(\dot{x}_{b}, \dot{x}_{a}\right)\right) .
\end{aligned}
$$

A direct calculation yields 


$$
\begin{aligned}
& \pi_{a \mu}\left(x_{c}, \dot{x}_{d}\right)= m_{a}\left(-\dot{x}_{a}^{2}\right)^{-1 / 2} \dot{x}_{a \mu} \\
&- \sum_{b \neq a} g_{a} g_{b} \frac{\partial F_{b a}\left(\dot{x}_{b}, \dot{x}_{a}\right)}{\partial \dot{x}_{a}^{\mu}} \\
& \times\left(1 / r_{a b} \sqrt{-\dot{x}_{b}^{2}}\right) \exp \left(-\mu r_{a b}\right), \\
& r_{a b}^{2}=x_{a b}^{2}-\left(\dot{x}_{b} x_{a b}\right)^{2} / \dot{x}_{b}^{2} .
\end{aligned}
$$

On the other hand, since the presence of the characteristic function $\Theta(\lambda-\eta, \lambda)$ makes the evaluation of $N_{b a}^{0}$ rather involved, it is more convenient to work it out in terms of its Fourier representation.

Taking into account that

$D_{\text {sym }}\left(\mu, x^{\mu} x_{\mu}\right)=4 \pi$ P.V. $\int_{\mathbb{R}^{4}} \frac{d^{4} k}{(2 \pi)^{4}} \frac{\exp (i k x)}{k^{2}+\mu^{2}}$,

we obtain

$$
N_{a b}^{0}=I_{a b}-I_{b a},
$$

where

$$
I_{a b}=4 \pi i \mathrm{P} . \mathrm{V} \cdot \int_{\mathbf{R}^{4}} \frac{d^{4} k}{(2 \pi)^{3}} \frac{\exp \left(i k x_{a b}\right)}{k^{2}+\mu^{2}} \frac{\delta\left(k \dot{x}_{b}\right)}{k \dot{x}_{a}},
$$

or, after a little calculation,

$$
I_{a b}=\frac{1}{\Lambda_{a b}} \int_{\mu_{a b}}^{1} d \eta \frac{\exp \left(-r_{a b} \mu \eta /\left(-\dot{x}_{b}^{2}\right)\right)}{\sqrt{\eta^{2}-u_{a b}^{2}}},
$$

where

$$
u_{a b}=\left(1-\left(\frac{\Lambda_{a b} z_{a b}}{r_{a b}}\right)^{2} \frac{1}{\left(-\dot{x}_{a b}^{2}\right)}\right)^{1 / 2},
$$

and the following Poincaré invariants have been introduced:

$$
z_{a b} \equiv \Lambda_{a b}^{-2}\left[-\dot{x}_{b}^{2}\left(x_{a b} \dot{x}_{a}\right)+\dot{x}_{a} \dot{x}_{b}\left(x_{a b} \dot{x}_{b}\right)\right]
$$

and

$$
\Lambda_{a b}^{2}=k_{a b}^{2}-\dot{x}_{a}^{2} \dot{x}_{b}^{2}, \quad k_{a b}=-\dot{x}_{a} \dot{x}_{b} .
$$

A similar result holds for $I_{b a}$.

Notice that, since $I_{a b}$ is Poincaré invariant, the calculations leading from $(5.10)$ to $(5.11)$ can be worked out in the rest frame of particle $b$, followed by the subsequent translation into covariant form.

The Liouville form (4.9), as well as the total linear and angular momenta, are determined to this order by $R_{a \mu}^{(2)}$ and $Q_{a \mu}^{(2)}$. Their explicit expressions can be found by direct substitution of (5.3), (5.8), and (5.10) into (5.7). The threedimensional formalism can also be developed by merely introducing the constraints (4.13).

\section{B. Wheeler-Feynman electrodynamics}

There is no doubt that the most widely known Fokkertype system is Wheeler-Feynman electrodynamics (WFE). First introduced by Tetrode ${ }^{22}$ and Fokker ${ }^{4}$ for two charges, its generalization to $N$ charges, ${ }^{5}$ supplemented by the perfect absorber theory, ${ }^{8}$ describes the whole classical electrodynamics, including the radiation reaction effects and the observed retarded interaction.

WFE corresponds to an action-at-a-distance vector interaction with vanishing mass parameter (i.e., $\mu=0$ ). Hence all the results hitherto obtained still hold.
Taking into account that

$\int \frac{d \eta}{\sqrt{A \eta^{2}+C}}=\frac{1}{\sqrt{A}} \ln \left(\sqrt{A \eta}+\sqrt{A \eta^{2}+C}\right), \quad A>0$,

we can express $N_{a b}^{0}$ in terms of elementary functions; thus obtaining

$$
N_{a b}^{0}=\frac{1}{2 \Lambda_{a b}} \ln \left(\frac{r_{a b}+\Lambda_{a b} z_{a b}\left(-\dot{x}_{b}^{2}\right)^{-1 / 2}}{r_{b a}+\Lambda_{a b} z_{b a}\left(-\dot{x}_{a}^{2}\right)^{-1 / 2}}\right),
$$

which, when introduced into (5.7), yields

$$
\begin{aligned}
R_{a \mu}^{(2)}= & m_{a} \frac{\dot{x}_{a \mu}}{\sqrt{-\dot{x}_{a}^{2}}}+\sum_{b \neq a} g_{a} g_{b}\left(\frac{\dot{x}_{a \mu}}{r_{a b} \sqrt{-\dot{x}_{b}^{2}}}\right. \\
& \left.+\frac{1}{2} \frac{k_{a b}}{2 \Lambda_{a b}} \frac{\partial}{\partial x_{a}^{\mu}} \ln \left(\frac{r_{a b}+\Lambda_{a b} z_{a b}\left(-\dot{x}_{b}^{2}\right)^{-1 / 2}}{r_{b a}+\Lambda_{a b} z_{b a}\left(-\dot{x}_{a}^{2}\right)^{-1 / 2}}\right)\right),
\end{aligned}
$$

$$
\begin{aligned}
Q_{a \mu}^{(2)}= & \frac{1}{2} \sum_{b \neq a} g_{a} g_{b} \\
& \times \frac{\partial}{\partial \dot{x}_{a}^{\mu}}\left[\frac{k_{a b}}{2 \Lambda_{a b}} \ln \left(\frac{r_{a b}+\Lambda_{a b} z_{a b}\left(-\dot{x}_{b}^{2}\right)^{-1 / 2}}{r_{b a}+\Lambda_{a b} z_{b a}\left(-\dot{x}_{a}^{2}\right)^{-1 / 2}}\right)\right] .
\end{aligned}
$$

By substituting these expressions into (4.15)-(4.17) we easily obtain the Hamiltonian and a set of canonical coordinates and momenta.

Furthermore, in order to compare with other results already known, the post-Newtonian approximation can be carried out. Approximating the canonical coordinates and momenta up to order $c^{-2}$, we obtain

$$
\begin{aligned}
\vec{q}_{a}= & \vec{x}_{a}-c^{-2} \sum_{b \neq a} g_{a} g_{b} \frac{1}{4 m_{a}} \frac{\partial}{\partial \vec{v}_{a}}\left(\frac{w_{a b}}{\left|\vec{x}_{a b}\right|}\right)+O\left(c^{-4}\right), \\
\vec{p}_{a}= & m_{a} \vec{v}_{a}+c^{-2}\left(\frac{1}{2} m_{a} v_{a}^{2} \vec{v}_{a}+\sum_{b \neq a} g_{a} g_{b} \frac{\vec{v}_{b}}{\left|\vec{x}_{a b}\right|}\right. \\
& \left.-\sum_{b \neq a} g_{a} g_{b} \frac{\partial}{\partial \vec{x}_{a}}\left(\frac{w_{a b}-w_{b a}}{\left|\vec{x}_{a b}\right|}\right)\right)+O\left(c^{-4}\right),
\end{aligned}
$$

where $w_{a b}=\vec{x}_{a b} \vec{v}_{a}$.

The simple form of the coordinates, up to this order of approximation, suggests the possibility of removing the $c^{-2}$ term by a canonical transformation with generating function,

$$
\begin{aligned}
& U(q, \tilde{p})=\sum_{a} \vec{q}_{a} \tilde{\vec{p}}_{a}+U_{2}(q, \tilde{p})+O\left(c^{-4}\right), \\
& U_{2}(q, \tilde{p})=c^{-2} \sum_{\substack{a, b=1 \\
a \neq b}}^{N} \frac{\vec{q}_{a b} \vec{v}_{a}}{4\left|\vec{q}_{a b}\right|}+O\left(c^{-4}\right),
\end{aligned}
$$

[where $\vec{v}_{a}=\overrightarrow{\vec{p}}_{a} / m_{a}+O\left(c^{-2}\right)$ must be understood in the $U_{2}$ term ], which leads to a new set of canonical coordinates and momenta, $\tilde{\vec{q}}_{a}$ and $\tilde{\vec{p}}_{a}$, that can be obtained by the well known expressions $^{21}$

$$
\tilde{\vec{q}}_{a}=\frac{\partial U(q, \tilde{\vec{p}})}{\partial \tilde{\vec{p}}_{a}}, \quad \vec{p}_{a}=\frac{\partial U(q, \tilde{p})}{\partial \vec{q}_{a}} .
$$

In this case 


$$
\begin{aligned}
& \tilde{\vec{q}}_{a}=\vec{x}_{a}+O\left(c^{-4}\right), \\
& \tilde{\vec{p}}_{a}=m_{a} \vec{v}_{a}+c^{-2}\left(\frac{1}{2} m_{a} v_{a}^{2} \vec{v}_{a}+\sum_{b \neq a} g_{a} g_{b} \frac{\vec{v}_{b}}{\left|\vec{x}_{a b}\right|}+\sum_{b \neq a} g_{a} g_{b} \frac{1}{2} \frac{\partial}{\partial \vec{x}_{a}}\left(\frac{w_{b a}}{\left|\vec{x}_{a b}\right|}\right)\right)+O\left(c^{-4}\right) .
\end{aligned}
$$

Hence, up to order $c^{-2}$, physical positions of particles can be taken as canonical coordinates. This result agrees with those given in Refs. 23 and 24.

In order to derive the order $c^{-4}$ terms for the canonical variables $\vec{q}_{a}$ and $\vec{p}_{a}$, correction terms must be added to Eqs. (5.17) and (5.18). Naming these correction terms $\vec{q}_{4 a}$ and $\vec{p}_{4 a}$, respectively, we have

$$
\begin{aligned}
& \vec{q}_{4 a}=c^{-4} \frac{1}{m_{a}}\left[\sum_{b \neq a} g_{b} g_{a} \frac{1}{1 b m_{b}} \frac{\partial}{\partial \vec{v}_{a}}\left(\mathscr{M}_{a b}-\mathscr{M}_{b a}\right)-\frac{v_{a}^{2}}{2} \sum_{b \neq a} \frac{g_{a} g_{b}}{m_{a}} \frac{\partial}{\partial \vec{v}_{a}}\left(\frac{w_{a b}}{\left|\vec{x}_{a b}\right|}\right)-\sum_{b \neq a} \frac{g_{a} g_{b}}{m_{a}}\left(\left(\frac{\partial}{\partial \vec{v}_{a}} \frac{w_{a b}}{\left|\vec{x}_{a b}\right|}\right) \vec{v}_{a}\right) \vec{v}_{a}\right], \\
& \vec{p}_{4 a}=c^{-4}\left[\frac{3}{8} m_{a} v_{a}^{4} \vec{v}_{a}+\sum_{b \neq a} g_{a} g_{b} \frac{1}{2\left|\vec{x}_{a b}\right|}\left(v_{a}^{2}-\frac{w_{b a}^{2}}{\left|\vec{x}_{a b}\right|^{2}}\right)-\sum_{b \neq a} g_{a} g_{b} \frac{1}{16} \frac{\partial}{\partial \vec{x}_{a}}\left(\mathscr{M}_{a b}-\mathscr{M}_{b a}\right)\right],
\end{aligned}
$$

where

$\mathscr{M}_{a b}(\vec{x}, \vec{v})=\frac{1}{\left|x_{a b}\right|^{3}}\left[\left(\vec{x}_{a b} \vec{v}_{b}\right)^{3}+\left(\vec{x}_{a b} \vec{v}_{a}\right)\left(\vec{x}_{a b} \vec{v}_{b}\right)^{2}\right]+\frac{1}{\left|\vec{x}_{a b}\right|}\left[\left(\vec{x}_{a b} \vec{v}_{b}\right)\left(3 \vec{v}_{b}^{2}-2 \vec{v}_{a} \vec{v}_{b}\right)+\left(\vec{x}_{a b} \vec{v}_{a}\right) \vec{v}_{b}^{2}\right]$

In order to compare these results with those given in Ref. 25, we carry out a canonical transformation with generating function

$$
U(q, \tilde{p})=\sum_{a} \vec{q}_{a} \tilde{\vec{p}}_{a}+U_{2}(q, \tilde{p})+U_{4}(q, \tilde{p})+O\left(c^{-4}\right)
$$

with

$$
\begin{aligned}
& U_{2}(q, \tilde{p})=c^{-2} \sum_{a, b} g_{a} g_{b} \frac{\vec{q}_{a b} \vec{v}_{a}}{4\left|\vec{q}_{a b}\right|}+O\left(c^{-6}\right), \\
& U_{4}(q, \tilde{p})=c^{-4} \sum_{a, b} g_{a} g_{b} \frac{1}{16 m_{a}} \mathscr{M}_{a b}(\tilde{q}, \vec{v})+O\left(c^{-6}\right),
\end{aligned}
$$

and

$$
\vec{v}_{a}=\frac{\tilde{\vec{p}}_{a}}{m_{a}}-c^{-2}\left[\frac{\tilde{p}_{a}^{2}}{2 m_{a}^{2}} \tilde{\vec{p}}_{a}+\sum_{b \neq a} g_{a} g_{b} \frac{\tilde{\vec{p}}_{b}}{m_{b}\left|\vec{q}_{a b}\right|}+\sum_{b \neq a} \frac{g_{a} g_{b}}{2 m_{b}} \frac{\partial}{\partial \vec{q}_{a}}\left(\frac{\vec{q}_{a b} \tilde{\vec{p}}_{b}}{\left|\vec{q}_{a b}\right|}\right)\right]+O\left(c^{-6}\right) .
$$

The final expressions for the canonical variables and momenta $\tilde{q}_{a}$ and $\tilde{p}_{a}$ up to order $c^{-4}$ coincide with the abovementioned results. ${ }^{25}$

\section{CONCLUSIONS AND OUTLOOK}

Let us briefly sketch the present state of affairs in those relativistic theories of directly interacting particles that are intended to relate the force acting on one particle to some classical field theory. This will help to understand the role claimed for the present paper and also for Ref. 9.

These theories start from a Fokker-type Lagrangian (A), which is nonlocal in time, and then the Euler equations are derived. The latter is a system of functional differential equations (B), which is nonpredictive in the Newtonian sense, because the particle position and velocities at a given time do not determine their future evolution. This seems to be a strong qualitative difference with regard to what is common in nonrelativistic physics. A way out is to accept that not all the solutions of (B) are "physically significant," but only those satisfying an additional requirement, namely, (C) the analytical dependence of particle world lines on some "small parameter" (either the inverse speed of light, the coupling constants, or the mass ratio).

The use of any of these criteria enables one to select a family of "physically significant" solutions of (B). Each of them can be parametrized by the particle positions and velocities at a given time, and hence satisfy a Newton-like set of equations of motion (D).

The path from (B) to (D) via (C) is conceptually simple when the inverse speed of light is taken as a "small parameter" (it can be carried over by merely iterating the substitution of lower orders of approximation into higher ones), and involves the use of more complex perturbative techniques, besides the theoretical framework of predictive relativistic mechanics, in the remaining two cases.

Finally, introducing some additional, although rather general, assumptions concerning the asymptotic conditions in the past and/or future infinity, a Hamiltonian formalism (E) can be set up. Apparently this canonical formalism has a relationship with the Fokker-type variational principle one has started from.

The main contribution of the present paper, and Ref. 9, too, consists of providing a Hamiltonian (presymplectic) 
formalism for Fokker-type Lagrangian systems. This enables us to introduce the analyticity condition directly in the Hamiltonian formalism, so that we can obtain a Hamiltonian formalism on a Newton-like phase space for the system of interacting particles. In this way, we abridge the path from (A) to (E), without losing the connection with the canonical formalism that seemed to underlie the Fokker variational principle.

We have finally specialized these general results to scalar and vector mesodynamics, ${ }^{6}$ and to Wheeler-Feynman symmetric electrodynamics, up to the first order in the coupling constants. Although the formalism has here been developed for a specific kind of theory, namely, relativisitc systems of directly interacting particles, its interest goes beyond this topic. Indeed, it could also be applied to nonlocal field theories (presumably with only a few extra technicalities not interfering with the core difficulty of a Lagrangian that is nonlocal in time). The canonical formalism so obtained would then allow one to proceed with a standard canonical quantization of nonlocal field theories and to add some new insight to other quantization procedures. ${ }^{26,27}$

\section{ACKNOWLEDGMENTS}

This work has been partially supported by CAICyT under Contract No. 0649-84. One of us (R. J.) wants to acknowledge the hospitality of the Universitat de Barcelona as well as the partial support of CONACYT (Mexico) and Ministerio de Educación y Ciencia, ICI (España).

\section{APPENDIX: FROM COVARIANT TO NONCOVARIANT NOTATION}

Here we give some formulas that are useful in translating expressions from covariant to noncovariant formalism, i.e., when the time fixation $x_{a}^{0}=t$ is introduced.

These formulas establish in what cases the operations (i) introducing the time fixations and (ii) differentiation commute or do not commute.

In general, we must deal with a Poincaré-invariant function $f\left(x_{c d}^{2}, x_{c d} \dot{x}_{e}, \dot{x}_{c} \dot{x}_{d}\right), c, d, e=1, \ldots, N$. Introducing the fixation, we have

$$
\tilde{f}\left(x_{c d}^{2}, x_{c d} \dot{x}_{e}, \dot{x}_{c} \dot{x}_{d}\right)=f\left(\left|\vec{x}_{c d}\right|^{2}, \vec{x}_{c d} \vec{v}_{e},-1+\vec{v}_{c} \vec{v}_{d}\right),
$$

where the notation given in Sec. IV has been used.

A short calculation proves that $\frac{\partial f}{\partial \vec{x}_{a}}=\sum_{c \neq a} 2 \vec{x}_{a c} \frac{\partial \tilde{f}}{\partial\left|\vec{x}_{a c}\right|^{2}}+\sum_{e, c} \vec{v}_{e} \frac{\partial \tilde{f}}{\partial\left(\vec{x}_{a c} \vec{v}_{e}\right)}=\frac{\partial \tilde{f}}{\partial \vec{x}_{a}}$

$\frac{\partial f}{\partial x_{a}^{0}}=-\sum_{e, c} \frac{\partial \tilde{f}}{\partial\left(\vec{x}_{a c} \vec{v}_{e}\right)}$,

$\frac{\partial f}{\partial \vec{x}_{a}}=\sum_{c \neq d} \vec{x}_{c d} \frac{\partial \tilde{f}}{\partial\left(\vec{x}_{c d} \vec{v}_{a}\right)}+\sum_{c} \vec{v}_{c} \frac{\partial \tilde{f}}{\partial\left(\vec{v}_{c} \vec{v}_{a}\right)}=\frac{\partial \tilde{f}}{\partial \vec{v}_{a}}$,

$\frac{\partial f}{\partial \dot{x}_{a}^{0}}=-\sum_{c} \frac{\partial \tilde{f}}{\partial\left(\vec{v}_{c} \vec{v}_{a}\right)}$.

'The Theory of Action at a Distance in Relativistic Dynamics, edited by $\mathbf{E}$. H. Kerner (Gordon and Breach, New York, 1972); Relativistic Action at a Distance, edited by J. Llosa; Lecture Notes in Physics, Vol. 162 (Springer, New York, 1982); Constraint Theory and Relativistic Dynamics, edited by G. Longhi and L. Lusanna (World Scientific, Singapore, 1987).

${ }^{2}$ P. Droz-Vincent, Rep. Math. Phys. 8, 79 (1975); F. Rohrlich, Lect. Notes Phys. 162, 190 (1982); N. Mukunda and E. C. G. Sudarshan, Phys. Rev. D 23, 2210 (1981); I. T. Todorov, Lect. Notes Phys. 162, 213 (1982); A. Komar, Phys. Rev. D 18, 1881 (1978); H. Sazdjian, Ann. Phys. (NY) 136, 136 (1981); R. Arens, Nuovo Cimento B 21, 395 (1974).

${ }^{3}$ L. Bel and J. Martin, Ann. Inst. H. Poincaré 22, 173 (1975); D. Dominici, J. Gomis, J. A. Lobo, and J. M. Pons, Nuovo Cimento B 61, 306 (1981). ${ }^{4}$ A. D. Fokker, Z. Phys. 58, 386 (1929).

${ }^{5}$ R. P. Feynman and J. A. Wheeler, Rev. Mod. Phys. 21, 425 (1949).

${ }^{'}$ P. Havas, Phys. Rev. 74, 456 (1948); 86, 974 (1952); H. Van Dam and E. P. Wigner, Phys. Rev. B 138, 1576 (1965); 142, 832 (1966); A. Katz, J. Math. Phys. 10, 1929 (1969): G. J. H. Burgers and H. Van Dam, ibid. 28, 677 (1987).

${ }^{7}$ W. N. Herman, J. Math. Phys. 26, 2769 (1985).

${ }^{8}$ R. P. Feynman and J. A. Wheeler, Rev. Mod. Phys. 17, 157 (1945).

${ }^{9}$ X. Jaén, R. Jaúregui, J. Llosa, and A. Molina, Phys. Rev. D 36, 2385 (1987).

${ }^{10}$ H. J. Babha, Phys. Rev. 70, 759 ( 1946).

'R. P. Gaida and V. I. Tretyak, Acta Phys. Pol. B 11, 504 (1980).

${ }^{12}$ F. J. Kennedy, J. Math. Phys. 10, 1349 (1969).

${ }^{13}$ R. Marnelius, Phys. Rev. D 10, 2535 (1974).

${ }^{14}$ L. Bel and J. Martin, Ann. Inst. H. Poincaré 22, 173 (1975).

${ }^{15}$ E. H. Kerner, J. Math. Phys. 3, 35 (1962).

${ }^{16} \mathrm{H}$. W. Woodcock and P. Havas, Phys. Rev. D 6, 3422 (1972).

${ }^{17}$ Synge, Proc. R. Soc. London 177, 118 (1941).

${ }^{18}$ L. Bel and J. Martin, Phys. Rev. D 8, 4347 (1973).

${ }^{19} \mathrm{~L}$. Bel and X. Fustero, Ann. Inst. H. Poincaré 25 (a), 411 (1976); (a) L. Bel and Z. H. Sirousse, Phys. Rev. D. 323128 (1985).

20J. W. Dettman and A. Schild, Phys. Rev. 95, 1057 (1954).

${ }^{21}$ F. Gantmacher, Lectures in Analytical Mechanics (MIR, Moscow, 1970).

${ }_{22} \mathrm{H}$. Tetrode, Z. Phys. 10, 317 (1922).

${ }^{23}$ J. Martin and J. L. Sanz, J. Math. Phys. 19, 780 (1978).

${ }^{24} \mathrm{~L}$. Bel and J. Martin, Ann. Inst. H. Poincaré 34, 231 (1980).

${ }^{25}$ X. Jaén, J. Llosa, and A. Molina, Phys. Rev. D 34, 2302 (1986).

${ }^{26} \mathrm{Ch}$. Hayashi, Prog. Theor. Phys. 10, 533 (1953).

${ }^{27}$ H. Hata, Phys. Lett. B 217, 438, 445 (1989). 